TITLE:

\title{
Similarities between implicit correction multigrid method and A- phi formulation in electromagnetic field analysis
}

\section{AUTHOR(S):}

Iwashita, Takeshi; Mifune, Takeshi; Shimasaki, Masaaki

\section{CITATION:}

Iwashita, Takeshi ... [et al]. Similarities between implicit correction multigrid method and A-phi formulation in electromagnetic field analysis. IEEE TRANSACTIONS ON MAGNETICS 2008, 44(6): 946-949

\section{ISSUE DATE:}

2008-06

URL:

http://hdl.handle.net/2433/84543

\section{RIGHT:}

(c) 2008 IEEE. Personal use of this material is permitted. However, permission to reprint/republish this material for advertising or promotional purposes or for creating new collective works for resale or redistribution to servers or lists, or to reuse any copyrighted component of this work in other works must be obtained from the IEEE. 


\title{
Similarities Between Implicit Correction Multigrid Method and A-phi Formulation in Electromagnetic Field Analysis
}

\author{
Takeshi Iwashita $^{1}$, Takeshi Mifune ${ }^{2}$, and Masaaki Shimasaki ${ }^{3}$ \\ ${ }^{1}$ Academic Center for Computing and Media Studies, Kyoto University, Kyoto 606-8501, Japan \\ ${ }^{2}$ Department of Electrical Engineering, Kyoto University, Kyoto 615-8510, Japan \\ ${ }^{3}$ Department of Applied Nuclear Engineering, Fukui University of Technology, Fukui 910-8505, Japan
}

\begin{abstract}
This paper proposes an implicit error correction method that corresponds to the explicit error correction methods, such as Hiptmair's hybrid smoother and the conventional multigrid method. The A-phi method can be seen as the implicit error correction method corresponding to the hybrid smoother. Numerical tests confirm that the A-phi method produces a similar correction effect on the error belonging to the kernel of the discrete curl operator as that of the hybrid smoother. Furthermore, this paper introduces an implicit correction multigrid method, which is the implicit error correction version of the conventional multigrid method. In this method, linear systems on all levels in a multigrid method are combined into a large linear system. This linear system is solved by an iterative solver, and any preconditioning techniques can be used. Numerical tests show that the proposed method involves coarse grid correction effects and achieves a convergence rate independent of the grid-size, thus confirming the effectiveness of the implicit error correction method.
\end{abstract}

Index Terms-A-phi method, hybrid smoother, implicit error correction, iterative method, multigrid method.

\section{INTRODUCTION}

$\mathbf{I}$ $\mathrm{N}$ a finite element electromagnetic field analysis, the most time-consuming part is the solution of the linear system of equations. Generally, a linear iterative solver such as the Incomplete Cholesky Conjugate Gradient (ICCG) method is used for this purpose [1]. It is well-known that the A-phi formulation gives a superior convergence rate to the A-formulation in the iterative solver [2], [3]. While several research papers have investigated the advantage of the A-phi method [4], [5], we provide another interpretation of its effectiveness in this paper.

First, we pay special attention to a similarity between Hiptmair's hybrid smoother [6] and a (two-level) multigrid method [7]. Both methods can be characterized by explicit error correction using a coefficient matrix that is different to the original one. Whereas the hybrid smoother uses the discrete gradient operator matrix, the multigrid method introduces coefficient matrices constructed on the coarse grids. Accordingly, we define a new class of such error correction techniques in iterative solvers, which we call the Explicit error correction method. Furthermore, corresponding to this explicit error correction method, we have proposed the Implicit error correction method. In Section II, we introduce the details of both these methods.

Next, it is shown that the A-phi method (formulation) can be seen as an implicit error correction method corresponding to Hiptmair's hybrid smoother. The authors have, therefore, suggested that the A-phi method has a similar effect to that of the hybrid smoother for the error that corresponds to the kernel of the discrete curl operator $K \operatorname{er}(\operatorname{curl})$ [8]. Whereas the hybrid smoother corrects these errors explicitly, the A-phi method introduces unknown variables for the electric scalar potential. $\mathrm{Nu}$ merical results show that the A-phi method reduces the error belonging to $\operatorname{Ker}(\mathrm{curl})$ in the conductive region more efficiently than the A-method.

Digital Object Identifier 10.1109/TMAG.2007.915076
Next, we introduce our new multigrid type iterative method, namely the Implicit correction multigrid method [8], which is an implicit error correction method corresponding to a conventional multigrid method. Numerical results of a 2-D finite difference analysis show that the implicit correction multigrid method achieves a convergence rate independent of the grid-size, which is the most important function of the multigrid method. Thus, the implicit error correction method can improve the condition of a coefficient matrix and can also be viewed as one of the preconditioning techniques.

\section{EXPLICIT ERROR CORRECTION METHOD AND IMPLICIT ERROR CORRECTION METHOD}

Let the linear system of equations to be solved be

$$
A x=b
$$

where $A$ is an $n \times n$ matrix, and $x$ and $b$ are $n$-dimensional vectors. When a linear system (1) is solved by an iterative solver, the explicit error correction method is often used. Let the current approximation for $x$ be $\tilde{x}$. The procedure used by the explicit error correction method is given by the following two steps. The first step is to determine error quantity $\tilde{y}$ using the following linear system:

$$
C y=d(\tilde{x}) .
$$

When the dimension of $y$ is denoted by $m$, the coefficient matrix $C$ is an $m \times m$ matrix. The $m$-dimensional vector $d$ is given by a function of the current approximation $\tilde{x}$. In general, the dimension $m$ is sufficiently smaller than $n$. Applying an iterative method such as a relaxation method or a direct solver to (2), we get an $m$-dimensional vector $\tilde{y}$, which is an approximation of $y$ or exactly $y$. The second step is given by the update of the approximation $\tilde{x}$

$$
\tilde{x}^{n e w} \leftarrow \tilde{x}+B \tilde{y}
$$


In this paper, we propose the implicit error correction method, in which the original linear system (1) and the (reduced dimensional) linear system for error correction (2) are combined. The implicit error correction method is derived as follows. First, while we pay attention to (3), the solution vector $x$ in (1) is replaced by $\hat{x}+B \hat{y}$, where $\hat{x}$ and $\hat{y}$ are $n$-dimensional and $m$-dimensional vectors, respectively. This means that the solution vector is described by $\hat{x}+B \hat{y}$. Consequently, we obtain

$$
A \hat{x}+A B \hat{y}=b
$$

instead of (1). Next, we consider the linear system for determining the error correction quantity (2). In most explicit error correction type methods, the linear system (2) is given by the restricted residual equation of (1). In this case, $d$ is written as a matrix-vector product $D r$, where $D$ is an $m \times n$ matrix and $r=b-A \tilde{x}$ is the residual vector. Then, in the implicit error correction method, we replace $d(\tilde{x})$ in (2) by $D(b-A \tilde{x})$. Accordingly, we get

$$
D A \tilde{x}+C y=D b .
$$

By rewriting vectors $\tilde{x}$ and $y$ by $\hat{x}$ and $\hat{y}$, respectively, we finally obtain the matrix form of the implicit error correction method as follows:

$$
\left(\begin{array}{cc}
A & A B \\
D A & C
\end{array}\right)\left(\begin{array}{l}
\hat{x} \\
\hat{y}
\end{array}\right)=\left(\begin{array}{c}
b \\
D b
\end{array}\right)
$$

In the proposed method, the linear system of (6) instead of (1) is solved by a (preconditioned) iterative method. The solution of the original linear system (1) is given by $\hat{x}+B \hat{y}$. It is expected that the iterative solution process produces the effect of the error correction implicitly. In other words, the linear system derived from the implicit error correction method (6) is expected to have an improved condition of the coefficient matrix over that of the original linear system (1).

\section{Relationship Between A-PHI Method AND HYBRID SMOOTHER}

In this paper, we deal with a quasi-static electromagnetic field problem. It is assumed that the analyzed domain $\Omega$ is a simply connected domain. For simplicity, we do not describe the effect of the boundary in the following. The basic equation based on the A-formulation is given by

$$
\nabla \times\left(\nu \nabla \times \vec{A}_{m}\right)+\sigma \frac{\partial \vec{A}_{m}}{\partial t}=\vec{J}_{0}
$$

where $\vec{A}_{m}, \nu, \sigma, t$, and $\vec{J}_{0}$ are the magnetic vector potential, the magnetic reluctivity, the electrical conductivity, time, and the exciting current, respectively. It is noted that $\nabla \cdot \vec{J}_{0}=0$ is satisfied. The basic equation based on the A-phi formulation is given by

$$
\begin{aligned}
\nabla \times\left(\nu \nabla \times \vec{A}_{m}^{\prime}\right)+\sigma \frac{\partial\left(\vec{A}_{m}^{\prime}+\nabla \phi\right)}{\partial t} & =\vec{J}_{0} \\
\nabla \cdot\left(\sigma \frac{\partial\left(\vec{A}_{m}^{\prime}+\nabla \phi\right)}{\partial t}\right) & =0
\end{aligned}
$$

where $\vec{A}_{m}^{\prime}$ is also the magnetic vector potential and $\phi$ is the electric scalar potential.

When a finite element discretization with edge elements and a backward time difference method are applied to (7), the resulting linear system of equations is given by

$$
K_{A} x_{A}=\left(C_{u}^{T} M_{\nu} C_{u}+\frac{M_{\sigma}}{\Delta t}\right) x_{A}=b_{A}
$$

where $\Delta t$ is the length of the time step, $x_{A}$ is the unknown vector that represents the magnetic vector potential to be calculated, and the right-hand side $b_{A}$ is determined by the previous value of the magnetic vector potential and the exciting current. When the number of edges and faces are denoted by $e$ and $f$, respectively, the matrix $C_{u}$ is an $f \times e$ matrix, which is given by the discrete curl operator. The $f \times f$ matrix $M_{\nu}$ and the $e \times e$ matrix $M_{\sigma}$ are given by

$$
\left[M_{\nu}\right]_{i j}=\int_{\Omega} \nu \vec{w}_{i}^{f} \cdot \vec{w}_{j}^{f} d V, \quad\left[M_{\sigma}\right]_{i j}=\int_{\Omega} \sigma \vec{w}_{i}^{e} \cdot \vec{w}_{j}^{e} d V
$$

where $\vec{w}^{f}$ and $\vec{w}^{e}$ are a face-element basis function and an edgeelement basis function, respectively.

In this paper, we consider the use of Hiptmair's hybrid smoother [6] in the A-method. Although the hybrid smoother consists of a normal Gauss-Seidel sweep and a special error correction process, the latter correction process is key to the method. Thus, we discuss only the error correction process in the following. When Hiptmair's hybrid smoother is applied to (10), the error correction procedure can be written in the form of an explicit error correction method as follows:

$$
\begin{aligned}
C & =\frac{1}{\Delta t} G^{T} M_{\sigma} G, \\
d\left(\tilde{x}_{A}\right) & =G^{T}\left(b_{A}-K_{A} \tilde{x}_{A}\right)
\end{aligned}
$$

in (2), and

$$
B=G
$$

where $\tilde{x}_{A}$ is the current approximation for $x_{A}$ and $G$ is a discrete gradient operator that satisfies $C_{u} G=0$. The matrix $C$ is also written as $G^{T} K_{A} G$. From (6), (10), (12), (13), and (14) and the relationship between $C_{u}$ and $G$, we obtain the linear system of the implicit error correction method corresponding to the hybrid smoother as follows:

$$
\begin{aligned}
& \left(\begin{array}{cc}
K_{A} & K_{A} G \\
G^{T} K_{A} & G^{T} K_{A} G
\end{array}\right)\left(\begin{array}{c}
\hat{x}_{A} \\
\hat{y}_{A}
\end{array}\right) \\
& =\left(\begin{array}{cc}
K_{A} & \frac{1}{\Delta t} M_{\sigma} G \\
\frac{1}{\Delta t} G^{T} M_{\sigma} & \frac{1}{\Delta t} G^{T} M_{\sigma} G
\end{array}\right)\left(\begin{array}{c}
\hat{x}_{A} \\
\hat{y}_{A}
\end{array}\right)=\left(\begin{array}{c}
b_{A} \\
G^{T} b_{A}
\end{array}\right) .
\end{aligned}
$$

The coefficient matrix in (15) is identical to the coefficient matrix arising from the finite element discretization of (8) and (9). Moreover, since the initial condition that $\hat{x}_{A}=x_{A}$ and $\hat{y}_{A}=0$ is normally set at $t=0, x_{A}=\hat{x}_{A}+G \hat{y}_{A}$ is satisfied in each time step. Consequently, the right-hand side of (15) is the same as the right-hand side derived from (8) and (9). Therefore, the linear system of the implicit error correction method that corresponds to the hybrid smoother (15) coincides with the linear system of equations derived from the A-phi method. Thus, the A-phi method can be regarded as the implicit version of the error correction of Hiptmair's hybrid smoother. 


\section{IMPLICIT CORRECTION MULTIGRID METHOD}

In this section, we introduce the implicit correction multigrid method, in which the linear systems of equations on all levels in a conventional multigrid method are combined and are solved simultaneously using a (preconditioned) iterative method. We first introduce the two-level implicit correction multigrid method. In a conventional two-level multigrid method, the coarse grid correction for (1) is given in the form of the explicit error correction method as follows:

$$
\begin{aligned}
C & =A^{H} \\
d(\tilde{x}) & =I_{h}^{H}(b-A \tilde{x})
\end{aligned}
$$

and

$$
B=I_{H}^{h}
$$

where $A^{H}$ is the coefficient matrix constructed on the coarse grid, and $I_{h}^{H}$ and $I_{H}^{h}$ are the restriction and prolongation operators, respectively. From (6), (16), (17), and (18), the linear system of the implicit correction multigrid method that corresponds to the coarse grid correction is given by

$$
\left(\begin{array}{cc}
A & A I_{H}^{h} \\
I_{h}^{H} A & A^{H}
\end{array}\right)\left(\begin{array}{c}
x \\
x^{H}
\end{array}\right)=\left(\begin{array}{c}
b \\
I_{h}^{H} b
\end{array}\right)
$$

where $x^{H}$ is an unknown vector with the dimension of the coarse grid.

The linear system for the multilevel implicit correction multigrid method is derived from the recursive application of the two-level implicit correction multigrid method to the original linear system (1). Here, we denote the linear system arising from the proposed method, for 0 to $k$ levels, by

$$
T^{k} x_{t}^{k}=b_{t}^{k} \text {. }
$$

When we solve (20) by means of the two-level implicit correction multigrid method, the resulting linear system is given by

$$
\left(\begin{array}{cc}
T^{k} & T^{k} I_{k+1}^{k} \\
I_{k}^{k+1} T^{k} & A^{k+1}
\end{array}\right)\left(\begin{array}{c}
x_{t}^{k} \\
x^{k+1}
\end{array}\right)=\left(\begin{array}{c}
b_{t}^{k} \\
I_{k}^{k+1} b_{t}^{k}
\end{array}\right)
$$

where $A^{k+1}$ and $x^{k+1}$ are the coefficient matrix and the unknown vector on the $k+1$ level, respectively, and $I_{k}^{k+1}$ and $I_{k+1}^{k}$ are the restriction and prolongation operators between the $k$ th and $(k+1)$ th levels, respectively. Applying (21) to (1) recursively, we finally obtain the linear system for the $l$-level implicit correction multigrid method as follows:

$$
\left(\begin{array}{ccc}
S^{0,0} & \ldots & S^{0, l} \\
\vdots & \ddots & \vdots \\
S^{l, 0} & \cdots & S^{l, l}
\end{array}\right)\left(\begin{array}{c}
x^{0} \\
\vdots \\
x^{l}
\end{array}\right)=\left(\begin{array}{c}
f^{0} \\
\vdots \\
f^{l}
\end{array}\right)
$$

where

$$
\begin{aligned}
S^{\alpha, \alpha} & =A^{\alpha} \\
S^{\alpha, \beta} & =A^{\alpha} I_{\alpha+1}^{\alpha} I_{\alpha+2}^{\alpha+1} \cdots I_{\beta}^{\beta-1}(\alpha<\beta) \\
S^{\alpha, \beta} & =I_{\alpha-1}^{\alpha} I_{\alpha-2}^{\alpha-1} \cdots I_{\beta}^{\beta+1} A^{\beta}(\alpha>\beta) \\
f^{\alpha} & =I_{\alpha-1}^{\alpha} I_{\alpha-2}^{\alpha-1} \cdots I_{0}^{1} b
\end{aligned}
$$

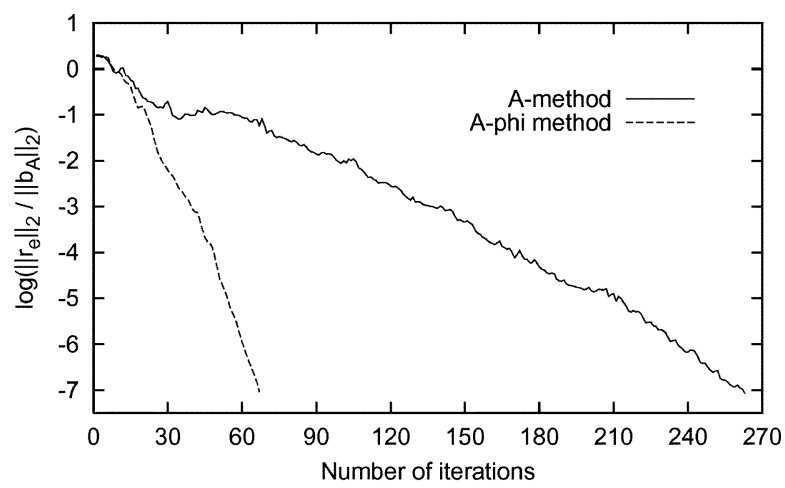

Fig. 1. Comparison of convergence between A-method and A-phi method.

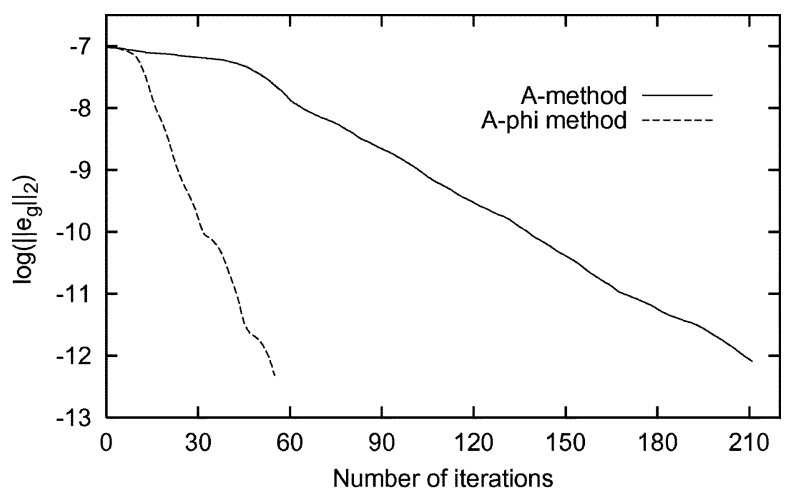

Fig. 2. Convergence behavior of the error of the kernel of the discrete curl operator in the conductive region.

$A^{0}=A$, and $f^{0}=b$. In the proposed method, the linear system (22) is solved using a preconditioned iterative method. The solution vector of the original linear system (1) is given by $x=x^{0}+\sum_{k=1}^{l}\left\{\left(\prod_{p=1}^{k} I_{p}^{p-1}\right) x^{k}\right\}$.

\section{NUMERICAL RESUltS AND DisCUSSION}

\section{A. Effect of the A-phi Method}

In this subsection, we examine the effect of the error correction in the A-phi method. The test problem is the Testing Electromagnetic Analysis Method (TEAM) workshop problem 10 [9]. The test model is discretized by using tetrahedra finite elements (Whitney elements), with the number of elements equal to 5968 , and $\Delta t$ set to $10^{-3}$ (s). The linear system in the first time step is solved using the ICCG method. Fig. 1 plots the comparison of convergence between the A-method and the A-phi method where $r_{e}$ is the residual of each linear system. This residual in the A-phi method is, however, given by $b_{A}-$ $K_{A}\left(\bar{x}_{A}+G \bar{y}_{A}\right)$ where $\bar{x}_{A}$ and $\bar{y}_{A}$ are the current approximations of $\hat{x}_{A}$ and $\hat{y}_{A}$, respectively. Fig. 1 shows the well-known advantage in convergence of the A-phi method, which has been explained by the superior condition number of the coefficient matrix [4]. Next, Fig. 2 shows the convergence behavior of the error $e_{g}$ of the kernel of the discrete curl operator $\operatorname{Ker}$ (curl) in the conductive region of the model. Whereas Hiptmair's hybrid smoother corrects this error explicitly, Fig. 2 indicates that the error $e_{g}$ can be efficiently removed in the A-phi method. Moreover, while we calculate the 2-norm ratio of $e_{g}$ with respect to the total error in the conductive region for the A-method, the ratio is higher than 0.9 in most of iterations. Thus, the $\operatorname{Ker}$ (curl) 


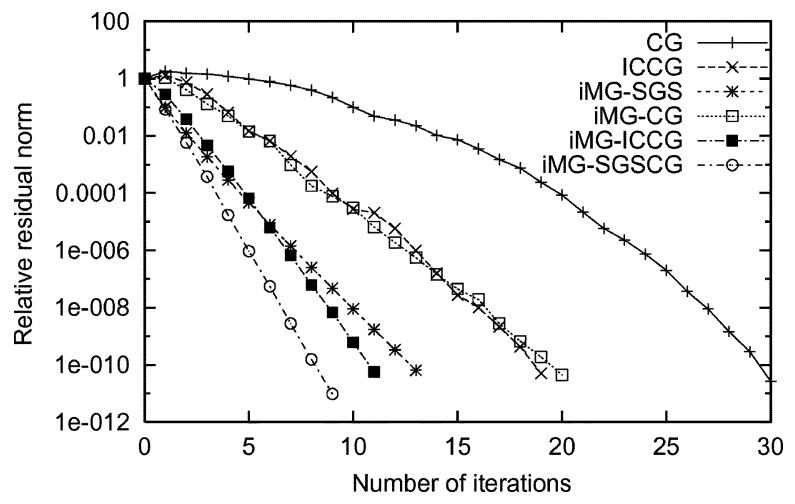

Fig. 3. Comparison of convergence behaviors $(15 \times 15$ grid $)$.

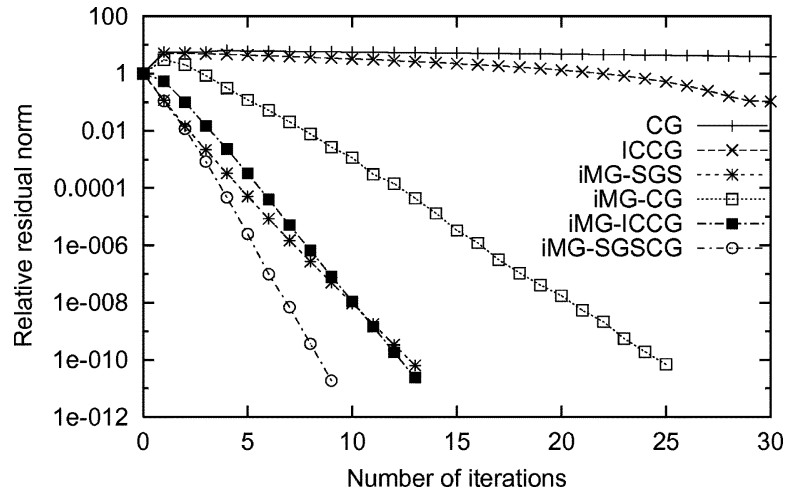

Fig. 4. Comparison of convergence behaviors $(127 \times 127$ grid $)$.

error causes the slow convergence rate in the A-method. Accordingly, the A-phi method has the effect of (implicit) error correction of $e_{g}$ as in the hybrid smoother, and therefore achieves the advantage in convergence.

\section{B. Effect of the Implicit Correction Multigrid Method}

In this subsection, we examine the effect of the implicit correction multigrid method. The test problem is given as a linear system of equations derived from a 2-D Poisson equation discretized by a five point difference method. Figs. 3 and 4 show a comparison of convergence behaviors, where "iMG" implies the implicit correction multigrid method. When the conjugate gradient (CG) or ICCG method is applied to the original linear system, the convergence rate declines rapidly as the problem size increases. On the other hand, when the linear system based on the implicit correction multigrid method is solved using the CG method, the number of iterations necessary for convergence does not really depend on the problem size. Since the procedure does not include any conventional multigrid process, this result indicates that the implicit correction multigrid method includes the coarse grid correction effect. Moreover, it is shown that the condition number of the coefficient matrix of the implicit multigrid method is improved from that of the original matrix 103 to 3.89 in the case of a $15 \times 15$ grid. Since the proposed method can be used together with various preconditioning techniques, it can potentially enlarge the areas of application of the multigrid method.

\section{Effectiveness of Implicit Error Correction Method}

Numerical results indicate that the implicit error correction method improves a condition of the coefficient matrix and at- tains a similar effect as the corresponding explicit error correction method. We now consider the condition under which the implicit error correction method works well. In general, an explicit error correction type method is introduced for correction of the error $e_{s}$ that satisfies $A e_{s} \simeq 0$. When $A$ is positive or semi-positive definite, these errors belong to a space spanned by the eigenvectors having small eigenvalues, which we denote by $V_{s}$. Accordingly, it is expected that the implicit error correction method can work well when the range of $B$ gives a good approximation of $V_{s}$. In this case, $D$ and $C$ can simply be set as $D=B^{T}$ and $C=B^{T} A B$ (Galerkin approximation), respectively, and then the coefficient matrix becomes singular. In our numerical tests, the implicit correction multigrid method shows good convergence performance under the above condition, even though the coefficient matrix on coarse levels can be constructed using other strategies.

\section{CONCLUSION}

This paper proposes an implicit error correction method that corresponds to the explicit error correction methods, such as Hiptmair's hybrid smoother and the coarse grid correction in a multigrid method. It is shown that the A-phi method can be regarded as an implicit error correction version of the hybrid smoother. Furthermore, numerical tests show that the A-phi method has a similar effect on the correction of the error of the kernel of the discrete curl operator as that of the hybrid smoother, which results in an advantage in convergence. Next, we introduce the implicit correction multigrid method by applying the implicit error correction concept to the multigrid method. It is shown that the proposed method achieves the convergence rate independent of the problem size. This result confirms the strong relationship between the explicit error correction method and the implicit error correction method and shows the effectiveness of the proposed method.

\section{REFERENCES}

[1] Y. Saad, Iterative Methods for Sparse Linear Systems, 2nd ed. Philadelphia, PA: SIAM, 2003.

[2] K. Fujiwara, T. Nakata, and H. Ohashi, "Improvement of convergence characteristic of ICCG method for the $A-\phi$ method using edge elements," IEEE Trans. Magn., vol. 32, no. 3, pp. 804-807, May 1996.

[3] R. D. Edlinger and O. Biro, "A joint vector and scalar potential formulation for driven high frequency problems using hybrid edge and nodal finite element," IEEE Trans. Microw. Theory Tech., vol. 44, no. 1, pp 15-23, Jan. 1996.

[4] H. Igarashi and T. Honma, "On convergence of ICCG applied to finite element equation for quasi-static fields," IEEE Trans. Magn., vol. 38, no. 2, pp. 565-568, Mar. 2002.

[5] B. Weiss and O. Biro, "On the convergence of transient eddy-current problems," IEEE Trans. Magn., vol. 40, no. 2, pp. 957-960, Mar. 2004.

[6] R. Hiptmair, "Multigrid method for Maxwell's equations," SIAM J. Numer. Anal., vol. 36, pp. 204-225, Dec. 1998.

[7] P. Wesseling, An Introduction to Multigrid Methods. New York: Wiley, 1992, Corrected Reprint. Philadelphia, PA: R.T. Edwards, Inc., 2004.

[8] T. Iwashita, T. Mifune, and M. Shimasaki, "New multigrid method: Basic concept of implicit correction multigrid method," (in Japanese) IPSJ Trans. Adv. Comput. Syst., vol. 48 (ACS18), pp. 1-10, May 2007.

[9] T. Nakata, N. Takahashi, and K. Fujiwara, "Summary of the results for benchmark problem 10 (steel plates around a coil)," Compel, vol. 11, pp. 335-344, 1992.
Manuscript received June 24, 2007. Corresponding author: T. Iwashita (e-mail: iwashita@media.kyoto-u.ac.jp). 